\title{
ASSESSING UNCERTAINTIES ASSOCIATED WITH DIGITAL ELEVATION MODELS FOR OBJECT BASED LANDSLIDE DELINATION
}

\author{
B. Feizizadeh ${ }^{a *}$, T. Blaschke ${ }^{b}$ \\ ${ }^{a}$ Dept. of Remote Sensing and GIS, University of Tabriz, Blvd 29 Bahman, Tabriz, Iran - Feizizadeh@tabrizu.ac.ir \\ ${ }^{\mathrm{b}}$ Dept. of Geoinformatics, University of Salzburg, 5020- Salzburg Austria - Thomas.Blaschke@ @bg.ac.at
}

KEY WORDS: Uncertainty analysis, DEM, Object based landslide mapping, Iran

\begin{abstract}
:
Digital elevation models (DEMs) are representations of topography with inherent errors that constitute uncertainty. DEMs data are often used in object based analyses without quantifying the effects of these errors. The main objective of this research is to establish a semi-automated object-based image analysis (OBIA) methodology for modelling uncertainty associated with DEMs when applied for locating landslides. In order to assess the uncertainty of DEMs, the Monte Carlo Simulation methodology was employed for evaluation of the effects of uncertainty on elevation and derived topographic parameters. The effect of DEM error is investigated, using stochastic conditional simulation to generate multiple equally likely representations of an actual terrain surface. Accordingly, distributional measures including accuracy surfaces, spatial autocorrelation indices, and variograms were also employed to quantify the magnitude and spatial pattern of the uncertainty. The semi-automated object based rule-sets for landslide delineation were developed based on two approaches a): without uncertainty assessing of DEMs and b): under applying uncertainty assessing of DEMs to examine the probable and possible uncertainties in delineating the landslides and measuring the improved accuracy after minimizing these associated uncertainties. The results of two approaches were validated using a landslide inventory database and very accurate GPS dataset. Results indicated very significant improvement in accuracy of results (> $28 \%$ ) when employing DEMs under uncertainty assessment. This research demonstrates how application of this methodology can address DEMs uncertainty, contributing to more responsible use of elevation and derived topographic parameters, and ultimately results obtained from their use.
\end{abstract}

\section{INTRODCTION}

Digital Elevation Models (DEMs) are known as key and fundamental data for variety of application in domain of geoscience. "DEMs represent the topography that drives surface flow and are arguably one of the more important data sources for deriving variables" (Wechsler, 2007, 1481). Due to the decrease in data and computer costs, increase in computing power as well as developing of geoscience technologies, the DEMs data has become increasingly available to spatial data users (Wechsler, 2007). The term of DEMs are often assumed to be accurate representations of the real-world terrain surfaces. However, as the name of "Model" is clearly indicates uncertainty is associated with any spatial modelling. Based on this observation it can be stated that DEMs are only an approximation representation of the true terrain surface (Oksanen, 2006; Venzin, 2013).The inherent uncertainty with DEMs may have significant impact on its accuracy and even some times leads inaccurate results (Feizizadeh and Blaschke 2014). Technically speaking, systematic and random errors have significant impacts on the accuracy of DEMs. These errors can range from elevation data acquisition to geospatial applications (Oksanen, 2006; Venzin, 2013). As already mentioned, DEMs frequently can be produced either based on remote sensing satellite images or topography dataset. However, both of these methods are subject to uncertainties due to measurement errors, interpolations of point data and digitization errors (Temme et al., 2009; Venzin, 2013). Wise (2000) and Reuter et al. (2009) classified uncertainties in DEMs into three category including a) artifact, blunders or gross error which can have huge impact on the derived land surface parameters, b) systematic errors which implies by data collection method and c) random errors or noise which are inherent in any DEMs and are caused by the measurement itself (i.e. single noise).It is critical to assess the inherent uncertainty associated with DEMs. Such an uncertainty analysis leads to reduce the errors, and its relative propagation of these errors on other derived parameters or models extracted from DEMs (Abd Aziz et al., 2012). In this regard, the accuracy of DEMs the Root Mean Square Error of Elevation (RMSE) is a most widely used technique which indicates the difference between the estimated and true value of DEMs products (Wise, 2000). However, according to Wechsler (2007) the RMSE method sometimes does not calculate an accurate assessment of how precisely each grid in a DEM represents topographical features. To solve this issue, number of researchers proposed spatial simulation methods for assessing the uncertainty of elevation estimates in each DEMs grid (Holmes et al., 2000; Carlisle, 2005; Wechsler and Kroll, 2006; Abd Aziz et al., 2012). The spatial simulation process analyses the spatial correlation in data to produce equiprobable estimates (realizations) of each particular grid in the DEMs. These realizations provide a range within which the true estimate lies and can be used to quantify the uncertainty at each DEM grid (Wechsler, 2007). Within this research we aimed to assess the inherent uncertainty associated with DEMs when applied to object based landslide mapping. Our research methodology make use of spatial analysis and statistical approaches such as Monte Carlo Simulation.

\section{STUDY AREA DATASET}

The study area was Ahar Chay- Basin which is sub-area of Urmai lake basin in northern-west of Iran. Geology setting of Ahar Chay- Basin is responsible for natural hazards and in particular to landslides. The landslide inventory database of study area indicated 32 landslide events which clearly acknowledge the potentiality of slopes in Ahar Chay- Basin for to landslides and mass movements (Feizizadeh and Blaschke 2012; Feizizadeh et al., 2012). Weathering, groundwater impacts, unstable slopes

\footnotetext{
* Corresponding author
} 
geological formation such as marlaceous and sedimentation combined with human impact issues such as land use/cover changes make the Ahar Chay- Basin prone to landslide and mass movements.

Within this research the semi-automated object-based approach was employed for delineating landslides. For this goal, DEMs derived from topography map 1:25 $000(10 \mathrm{~m})$ was used to extract morphometric features. In order to measure the improved accuracy under applying uncertainty analysis, the DEMs was employed in two approaches. Within the first approach the row DEMs data was used as base of landslide delineation by applying OBIA techniques. While, in the second approach the uncertainty analysis was performed on DEMs data and the accuracy of DEMs was improved by minimizing the associated uncertainties. Accordingly, the revised DEMs was employed for object based landslide delineation. To delineate landslides in object based manner, the information gathered from different parameter features provided by OBIA and landslide inventory dataset as thematic GIS layers.

\section{METHDOLOGY}

In order to determine the uncertainty associated with DEMs, it is necessary to compute the error at first step. Within this research the error associated with DEMs was computed as following: -Error

$\left(x_{\circ}\right) e\left(x_{\circ}\right)=Z\left(x_{0}\right)-\hat{Z}\left(x_{\circ}\right)$

$\mathrm{e}\left(\mathrm{x}_{\circ}\right)$ is error in location

$\mathrm{Z}\left(\mathrm{x}_{\circ}\right)$ is the accurate number

$\hat{\mathrm{Z}}\left(\mathrm{x}_{0}\right)$ is the calculated amount

- Bias

Bias is fundamental model which can be calculated from average of error based on statistical approaches as following:

$\mathrm{ME}=\frac{1}{\mathrm{n}} \sum_{\mathrm{i}=1}^{\mathrm{n}}\left(\widehat{\mathrm{Z}}\left(\mathrm{x}_{\mathrm{i}}\right)-\mathrm{Z}\left(\mathrm{x}_{\mathrm{o}_{\mathrm{i}}}\right)\right)$

Precision

Precision or true value is also another fundamental model which can be calculated by measuring the error distribution from average as following:

$$
\mathrm{STD}=\sqrt{\frac{\sum_{\mathrm{i}=1}^{\mathrm{n}}\left(\overline{\mathrm{e}}_{\mathrm{i}}-\hat{\mathrm{e}}_{\mathrm{i}}\right)^{2}}{\mathrm{n}-1}}
$$

Accuracy in DEMs is also known as sum of precision and unBias. According to this, the difference between the estimated and true value is often available for final DEMs products and indicated by the RMSE (Wise, 2000):

$$
\text { RMSE }=\sqrt{\frac{\sum_{\mathrm{i}=1}^{\mathrm{n}}\left(\mathrm{z}\left(\mathrm{s}_{\mathrm{i}}\right)-\hat{\mathrm{z}}_{\mathrm{REF}}\left(\mathrm{s}_{\mathrm{i}}\right)\right)^{2}}{\mathrm{n}}}
$$

where $\mathrm{z}\left(\mathrm{s}_{\mathrm{i}}\right)$ refers to the $i^{\text {th }}$ interpolated elevation, $\hat{\mathrm{z}}_{\mathrm{REF}}\left(\mathrm{s}_{\mathrm{i}}\right)$ refers to the $i^{\text {th }}$ known or measured elevation and $\mathrm{n}$ is the number of samples.

However, it is believed that measuring the error associated with DEMs is not easy task. Sometimes small error can have a significant impact on accuracy of DEMs, whereas large errors may cancel out in other cases. Even though DEMs with a high spatial resolution are often interpreted as less uncertain, in order to model this uncertainty our research also make use the advantage of Monte Carlo Simulation which is known as effective approach for uncertainty analysis.

\subsection{MONTE CARLO SIMULATION ON DEM}

Monte Carlo simulation approach is one the most widely used approaches for uncertainty analysis. This methods has been employed by many researchers to evaluate error associated with GIS datasets (Wechsler and Charles, 2006). In order to make of Monte Carlo Simulation for DEMs uncertainty analysis, it is required to recognize DEMs as only one possible realization of the true elevation surface. Within this approach multiple simulations can be used to quantify DEMs uncertainty through evaluation of statistics associated with a distribution of realizations (Venzin, 2013). Based on the Monte Carlo approach, the error propagation problem can be formulated as follows:

$U=g\left(A_{1, \ldots, A_{n}}\right)$

Where $U$ is the output of a GIS operation $g$ on $n$ different inputs $A_{i}$. According to this equation, the objective of uncertainty analysis is to compute the uncertainty in the output $\mathrm{U}$, given the operation $g$ and the uncertain input $A_{i}$. The Monte Carlo method aims to compute $\mathrm{U}$ multiple times with inputs $a_{i}$ which are randomly sampled from their joint probability distribution of $A_{i}$ (Venzin, 2013). According to Temme et al. (2009) the Monte Carlo Simulation consists of the following steps:

I. Repeat $\mathrm{N}$ times:

a) Generate a random realization $a_{i}$

b) For each $a_{i}$, compute and store the output $\mathrm{u}=\left(a_{i}, \ldots, a_{n}\right)$

II. Compute and store sample statistics from the $N$ output $u$

In order to generate a random sample $a_{i}$ of $A_{i}$, a pseudo random number generator is required (Van Niel and Laffan, 2003). Due to the joint probability distribution, higher number of realizations $\mathrm{N}$ leads to obtain more stable results. Brown and Heuvelink (2006) indicates that the accuracy of Monte Carlo approach is proportional to the square root of the number of runs $\mathrm{N}$. This means that any degree of accuracy can be reached with a large enough number of realizations $\mathrm{N}$, but also that the computation time of an uncertainty analysis quadruples if we want to double the accuracy. Each Monte Carlo Simulation run results in one realization. The sum of the realizations can be used to derive several statistical estimators such as the standard deviation describing the uncertainty of the computed product (Venzin, 2013). The minimum amount of $\mathrm{N}$ should be at least 100 and that less than 20 simulations are insufficient. Oksanen (2006) also highlighted that the minimum amount of simulation has to be about 500. Within this research we considered 1000 runs for assessing uncertainty associated with DEMs. Accordingly in order to create this distribution a number $(\mathrm{N})$ of random error fields are generated where each cell represents the possible error at a co-located elevation. Each random field is added to the DEMs generating a new realization of the elevation surface (Wechsler and Charles, 2006).

\subsection{OBJECT BASED LANDLIDE DELEIANTION}

Within this research we follow methodology proposed by Blaschke et al. (2014) for object-based landslide mapping. This methodology makes use of integrated approach of spectral and spatial characteristics for modelling and delineating landslides. As first step of this methodology, the multi-resolution segmentation process was performed to create objects which were analysed with respect to their spatial, spectral, and textural parameters. The segmentation was performed using five scale parameters $(5,7,8,9$ and 10$)$, with the same shape $(0.3)$ and compactness $(0.5)$ factors. Then, the data were parameterized according to the specific requirements for mapping landslides 
and incorporated into a multi-scale optimization routine (Stumpf et al., 2011). In this way we aimed to integrate the spectral, spatial, and morphometric characteristics of landslides. Slope and flow directions were derived from the DEMs. The landslide inventory dataset were used as samples to develop object based rule-set for landslide mapping. An analysis of the landslide inventory map indicated that all of the 32 landslides objects occurred at high elevations $(>1450 \mathrm{~m}$ ) in areas with slopes (> 7 $\%$ ). Based on this dataset, whole objects were then classified into either "potential landslide" or "no landslide". According to the previous works regarding geomorphology of Ahar Chay- Basin, most of the known landslides are categorized as rotational landslides (Feizizadeh and Blaschke 2012, Feizizadeh et al., 2012). The morphological characteristics of these rotational slides exhibit abrupt changes in slope morphology, with concavity in the depletion zone of the landslide and convexity in the accumulation zone (Akbarimehr, 2013). In doing so, to delineate landslides object based techniques including mean, standard deviation, GLCM texture parameters (GLCM Contrast, GLCM Correlation, GLCM Entropy, GLCM Mean, GLCM Standard Devotion) were computed as base of rule-sets. Landslide inventory data were also employed as sampling data for obtaining thresholds.

\section{RESULTS}

Results of obtained landslide objects for both approaches are represented in figure 1. Based on the results of first approach (landside delineation using row DEMs) totally 44 landslide object were identified and the area affected by landslides were measured approximately 822 hectares. While, the identified landslide objects from the second approach by employing revised DEMs was counted 36 with area about 484 hectares.

In order to validate results, the accuracy assessment process was performed by applying known landslide locations within the study area. It is believed that, accuracy assessment is fundamental step in object based landside delineation and the quality of a detected landslide inventory depends on its accuracy (Moine et al., 2009; Blaschke et al., 2014). According to Moine et al. (2009) defining the accuracy of a landslide inventory is not straightforward, and standards do not exist. However, to evaluate the accuracy of our results, the OBIA-derived landslides were compared to an existing landslide inventory map of the study area. This landslide inventory database was used as a reference to implement the accuracy assessment. Results of accuracy assessment indicated the overall accuracy of $68.04 \%$ for landslide map delineated from row DEMs. While this overall accuracy significantly improved to $96.92 \%$ when the revised DEMs was employed as base of landslide delineation rule-sets. Figure 2 represent comparison between known landslides and landslides delineated from both DEMs.

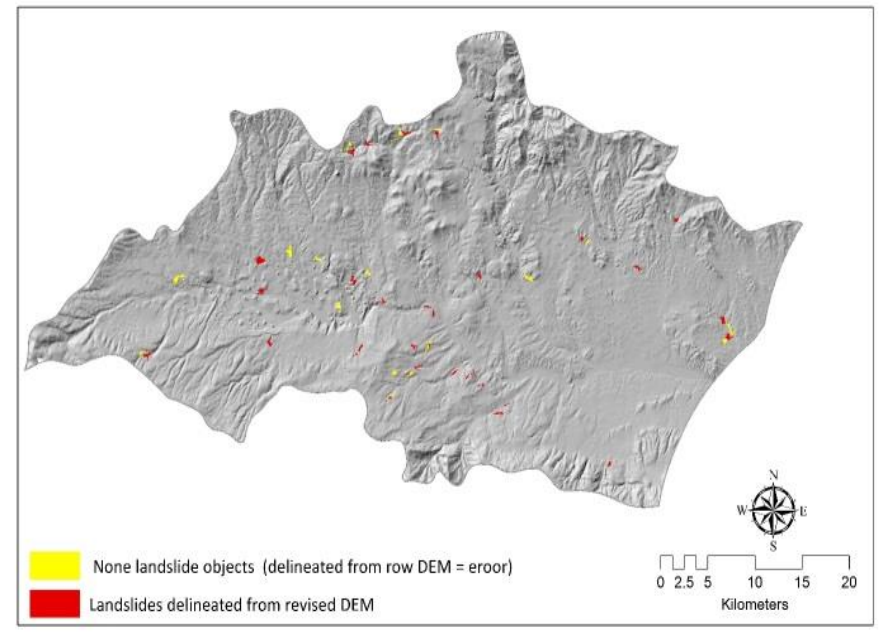

Figure 1. Landslides delineated from both DEMs

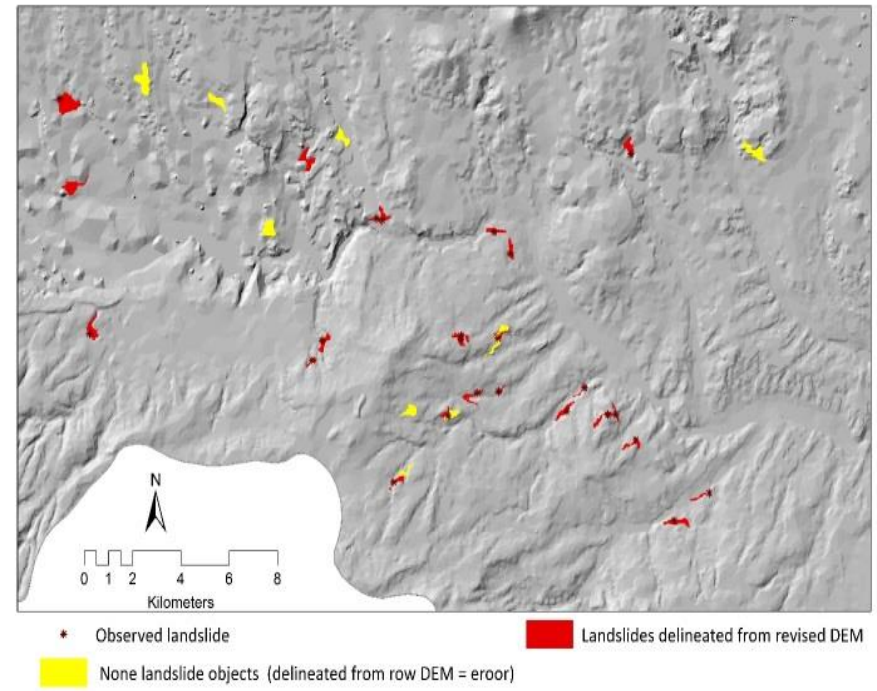

Figure 2. Results of accuracy assessment

\section{REFERENCES}

Akbarimehr, M., Motagh, M., Haghshenas-Haghighi, M., 2013. Slope Stability Assessment of the Sarcheshmeh Landslide, Northeast Iran, Investigated Using InSAR and GPS Observations". Remote Sensing. vol. 5, pp. 3681-3700.

Abd Aziz, A., Steward, B.L., Kaleita,A., Karkee, M., 2012. Assessing the effects of DEM uncertainty on erosion rate estimation in agricultural filed, journal of agriculture and biosystem engraining, 55(3): 785-798.

Blaschke, T., Feizizadeh, B., Holbling, D., 2014.Object-Based Image Analysis and Digital Terrain Analysis for Locating Landslides in the Urmia Lake Basin, Iran, IEEE Journal of Selected Topics in Applied Erath Observation and Remote Sensing, 7( 12), 4806-4817.

Carlisle, B. H., 2005. Modeling the spatial distribution of DEM error. Trans. GIS 9(4): 521-540.

Feizizadeh, B., Blaschke, T., 2014. Uncertainty analysis of GISMulticriteria based landslide susceptibility mapping. International Journal of Geographic Information Science, DOI:10.1080/13658816.2013.869821. 
Feizizadeh, B., Blaschke, T., 2012. GIS-Multicriteria Decision Analysis for landslide susceptibility mapping: comparing three methods for the Urmia lake basin, Iran. Natural Hazards, vol. 65 (3), pp. 2105-2128.

Feizizadeh, B., Blaschke, T., Nazmfar, 2012. GIS-based ordered weighted averaging and Dempster Shafer methods for landslide susceptibility mapping in Urmia lake Basin, Iran". International Journal of Digital Earth, DOI:10.1080/17538947.2012.749950.

Holmes, K. W., Chadwick, O. A., Kyriakidis, P. C., 2000. Error in a USGS 30-meter digital elevation model and its impact on terrain modelling. J. Hydrol. 233(1-4): 154-173.

Lu, P., Stumpf, A., Kerle, N., Casagli, N., 2011. Object-Oriented Change Detection for Landslide Rapid Mapping. IEEE Geoscience and Remote Sensing Letters, vol. 8 (4), pp. 701-705, 2011.

Moine, M., Puissant, A., Malet J. P., 2009. Detection of landslides from aerial and satellite images with a semiautomatic method. Application to the Barcelonnette basin (Alpes-de-Haute-Provence, France). In: J. P. Malet, A. Remaitre and T. Bogaard (Eds.), "Landslide Processes: From Geomorphological Mapping to Dynamic Modelling”, CERG, Strasbourg, France, pp. 63-68, 2009

Oksanen, J., 2006. Digital elevation model error in terrain analysis. PhD thesis, Faculty of Science, University of Helsinki.

Reuter, H.I., Hengl, T., Gessler, P., Soille, P., 2009. Preparation of DEMs for Geomorphometric Analysis. In: Tomislav, H\& Hannes, I.R (eds) Developments in soil science. Chapter 4, pages $87-120$.

Temme, A., Heuvelink, G., Schoorl, J., and Claessens, L., 2009. Geostatistical Simulation and Error Propagation in Geomorphometry. In Hengl, T. and Reuter, H. I., editors, Geomorphometry Concepts, Sof tware, Applications, volume 33 of Developments in Soil Science, chapter 5, pages 121-140.

Venzin., 2013, Analyzing the Impact of High Resolution DEM Uncertainty on Hydrological Models Using a Parallel Computing Approach, Master Thesis, Department of Geography, GIScience, Geocomputation, University of Zurich.

Wechsler, S.P., Charles, N.K., 2006. Quantifying DEM uncertainty and its effect on topographic parameters, Photogrammetric Engineering \& Remote Sensing, 72, No. 9, September 2006, pp. 1081.

Wechsler, S. P., 2007. Uncertainties associated with digital elevation models for hydrologic applications: a review. Hydrology and Earth System Sciences, 11(4):1481-1500.

Wise, S., 2000. Assessing the quality for hydrological applications of digital elevation models derived from contours. Hydrological Processes, 14(11-12):1909-1929. 